\title{
Percepciones sobre el aprendizaje del diseño gráfico en los estudios universitarios de Periodismo en España: contenidos, utilidad y nivel de capacitación
}

\author{
Fernando SuÁrez CARBAllo \\ Universidad Pontificia de Salamanca \\ fsuarezca@upsa.es \\ Juan Ramón MARTín SAN ROMÁN \\ Universidad Pontificia de Salamanca \\ jrmartinsa@upsa.es
}

Recibido: 1 de septiembre de 2014

Aceptado: 10 de marzo de 2015

\begin{abstract}
Resumen
El presente estudio pretende examinar la situación del diseño gráfico en la formación de los estudiantes universitarios de Periodismo en España a partir de las percepciones de profesionales y docentes especializados en esta área. La investigación trata de evaluar la presencia de estos conocimientos desde tres bloques temáticos principales (contenidos, utilidad y nivel de capacitación), con objeto de revelar en qué medida las competencias ofrecidas por los planes de estudio efectivamente satisfacen las exigencias de la profesión. A partir del análisis de estos tres parámetros mediante una metodología de encuesta cuantitativa, el artículo sugiere que tales estudios se prevén insuficientes en relación a los requisitos demandados por la realidad laboral.
\end{abstract}

Palabras clave: diseño gráfico, formación, periodismo, universidad, estudios.

\section{Perceptions about learning graphic design in Journalism university studies in Spain: contents, utility and training level}

\begin{abstract}
This study aims to examine the situation of graphic design in the Journalism university students training in Spain through the perceptions of professionals and professors specializing in this area. The research tries to evaluate the presence of this field according to three main thematic viewpoints (contents, utility and training level), in order to discover how the abilities offered by these programs respond to the professional requirements. From the analysis of these parameters with a quantitative survey methodology, the article concludes that these studies are insufficient compared to the work demands.

Keywords: graphic design, training, journalism, university, studies.

\section{Referencia normalizada}

SUÁREZ CARBALLO, Fernando y MARTÍN SAN ROMÁN, Juan Ramón (2015): "Percepciones sobre el aprendizaje del diseño gráfico en los estudios universitarios de Periodismo en España: contenidos, utilidad y nivel de capacitación”. Estudios sobre el Mensaje Periodístico. Vol. 21, Núm. 2 (julio-diciembre), págs.: 1255-1273. Madrid, Servicio de Publicaciones de la Universidad Complutense.
\end{abstract}

Sumario: 1. Introducción y estado de la cuestión. 2. Metodología; 2.1. Objetivo e hipótesis de trabajo; 2.2. Estudio exploratorio; 2.3. Sujetos; 2.3.1. Perfil docente; 2.3.2. Perfil profesional; 2.4. Instrumento de recogida de datos; 2.5. Procedimiento de investigación. 3. Resultados y discusión; 3.1. Bloque información general (perfil de la muestra); 3.2. Bloque contenidos; 3.3. Bloque utilidad; 3.3.1. Sobre el volumen necesario de conocimientos de diseño gráfico; 3.3.2. Sobre la importancia atribuida por los estudiantes; 3.3.3. Sobre el número de alumnos que se dedican al diseño gráfico; 3.4. Bloque capacitación; 3.4.1. Sobre el nivel de preparación en diseño gráfico de la carrera; 3.4.2. Sobre el rendimiento de los estudiantes en diseño gráfico. 4. Conclusiones. 5. Limitaciones y líneas futuras de investigación. 6 . Referencias bibliográficas. 


\section{Introducción y estado de la cuestión}

Entendido como una actividad comunicativa, industrial, proyectual y funcional (González Solas, 2001; Rom, 2002), el diseño gráfico es comúnmente considerado como uno de los elementos que intervienen de forma decisiva en la producción del mensaje periodístico (desde una perspectiva multidisciplinar y asociado a vehículos informativos de diversa naturaleza: impreso, audiovisual, interactivo o electrónico). Al mismo tiempo, su aprendizaje ha estado tradicionalmente ligado a los programas universitarios en el ámbito de la comunicación y, en concreto, a la rama de Periodismo, con mayor o menor intensidad en cada uno de los diferentes planes de estudio.

La vertiente formal, es, en efecto, un aspecto indisociable del propio mensaje. Aludiendo concretamente al ámbito del periodismo impreso, Casasús describe un diario como "un medio de comunicación de masas con una materia significante de orden visual, con una infraestructura material y con unas series informacionales lingüísticas, paralingüísticas, icónicas y no-lingüísticas" (1998: 96), que determinan su código. En efecto, un diario se define a partir de una serie de elementos de diversa naturaleza (entre los que figura el diseño gráfico) que, coordinados entre sí y en tanto que miembros de una estrategia comunicativa común, derivan y se concretan en el diario como producto informativo. También en relación a la envergadura del Diseño Periodístico (concretamente), Contreras y San Nicolás (2002: 58) apuntan que no es, "en ningún momento, independiente de los procesos propios del periodismo", y García Yruela (1991: 102) insiste en esta premisa al afirmar que "el diseño (entendido, pues, como la formalización de la información o como parte de su vertiente 'expresiva') es parte sustancial e indisociable del hecho informativo, y de él (y de su vínculo con el contenido mismo) depende la coherencia del medio impreso y su credibilidad".

Aunque muchas reflexiones, como las anteriores, aluden específicamente a la contribución del diseño gráfico en el ámbito de las publicaciones informativas impresas de periodicidad diaria, las definiciones bien pueden servir para ilustrar su incidencia en otros medios de comunicación ${ }^{1}$ : en efecto, la participación del diseño gráfico en la industria periodística es mucho más rica y afecta, con la misma envergadura, a un mayor número de sectores, como las revistas impresas, la infografía, las plataformas audiovisuales, la web, los más modernos dispositivos electrónicos, determinados materiales corporativos y, en definitiva, cualquier iniciativa periodística que emplee la imagen como vehículo de comunicación. Todos ellos acuden al diseño gráfico no solo para formalizar sus contenidos sino, también, en tanto que una de sus misiones más inmediatas, para fortalecer la eficacia del mensaje. Como argumenta Frechette (2013), "bad graphic design can damage perceptions about your credibility. It can make your content harder to understand and render your work less appealing".

En resumen, el diseño gráfico debe ser considerado como un núcleo inextricable

1 En relación al capítulo visual, el periodismo impreso es, probablemente (junto al género infográfico), el ámbito de mayor tradición científica y académica. No obstante, la presente investigación insiste en huir de esta restricción y busca trasladar la influencia del diseño gráfico a cualquier ámbito periodístico en el que la imagen tenga una presencia importante (exceptuando, básicamente, aquellos campos que prescindan por completo de este elemento, como la radio). 
del mensaje periodístico; como sostienen González Díez y Núñez Romero (2009: 86), "el diseño no es decoración, ni distracción, sino parte de la tarea de comunicación: una parte que debemos considerar constantemente". Y, por tanto, para la ejecución eficaz de estas tareas, el diseño debe enmarcarse dentro de una formación periodística rigurosa e integral. En este sentido, en relación al viejo debate sobre la procedencia y la formación de los diseñadores que ejercen su labor en el ámbito del periodismo (facultades de Bellas Artes y escuelas de Diseño han sido frecuentes canteras de los departamentos gráficos), Benito (2011) señala que estos profesionales deben ser, ante todo, periodistas, "y, por lo tanto, la formación debe ser fundamentalmente periodística, sin que ello signifique que tengan que descuidarse otras facetas". Así pues, como insiste De Pablos (2003: 50), es imprescindible que estos conocimientos se incorporen de forma natural a los programas formativos: en su opinión, "los profesionales, sean del sector informativo o del área productiva, cada día tendrán que disponer de mayores conocimientos y habilidades tecnológicas"2.

Efectivamente, en un entorno cambiante, en el que la propia tecnología remueve modas, comportamientos o demandas sociales y, al mismo tiempo, redefine constantemente las rutinas profesionales del periodismo, los conocimientos en diseño gráfico se antojan imprescindibles para una formación global del futuro periodista.

Es indudable que los actuales perfiles profesionales en el ámbito de la comunicación requieren de un programa innovador capaz de formar de manera integral a los nuevos profesionales que se están precisando en la nueva era de la información en la que estamos inmersos (González Díez y Núñez-Romero, 2009: 75).

La propia ANECA (2005) reconoce el diseño como una de las competencias esenciales en la profesión periodística. Como recuerdan González Díez y Núñez-Romero (2009), en un estudio que aborda la enseñanza universitaria del diseño periodístico como un vehículo para la inserción profesional, el rol de diseñador está destacado en el Libro Blanco del Grado de Periodismo como el primero de los cuatro posibles perfiles profesionales, principalmente:

"Redactor/a de información periodística en cualquier tipo de soporte: Profesional del periodismo en cualquier soporte mediático tradicional o electrónico que desarrolla su actividad mediante los géneros creando contenidos periodísticos. Comprende las tareas de redactor, reportero, presentador y director de uno o de varios de ellos, incluido el diseño, escritura y ejecución de guiones de reportajes y documentales audiovisuales o multimedia" (ANECA, 2005: 191).

Así pues, los anteriores párrafos parecen revelar un consenso claro entre profesionales, docentes y los propios organismos de ordenación académica sobre la relevancia de los conocimientos de comunicación visual en las funciones del periodista y sobre la responsabilidad de la universidad en su gestión educativa. Como afirman Car-

2 Tecnología y diseño gráfico son conceptos que han compartido escenario de forma habitual en los planes de estudio y cuyo vínculo, aun siendo parcelas suficientemente autónomas, es difícil quebrar en la tarea periodística. Como asegura Pérez Cuadrado (2009), la confusión parece haber sido alimentada por la antigua denominación de ciertas asignaturas (véase Tecnología de la Información). En el argumento de De Pablos (2003), el contexto sugiere que deben considerarse simultáneamente. 
doso et al. (2011), esta institución "tiene una papel esencial en la formación de los futuros profesionales que no puede ser disociado de la realidad empresarial", expectativas que, según Salaverría y Barrera (2009), se han ido progresivamente cumpliendo con el paso del tiempo: "nowadays there is a fairer balance between cultural background, communication theory and professional training in schools, and students enjoy a wider range of facilities". Y, pese a ello, en la práctica, muchas voces se lamentan de la escasa relevancia que presentan las materias de diseño gráfico en los planes de estudio de Periodismo. González Díez y Núñez-Romero (2009: 75) se hacen eco de este vacío:

Tradicionalmente, las Facultades de Ciencias de la Información han vivido de espaldas al Diseño, siendo éste una actividad casi marginal en las mismas. Con la adaptación al Espacio Europeo de Educación Superior (EEES) -es decir, Bolonia- la situación, en muchos casos, no va a mejorar, pues muchas universidades ni siquiera contemplan la docencia de asignaturas vinculadas al Diseño y las más incluyen muy pocas asignaturas vinculadas a la materia objeto de estudio.

Como sugieren estos autores, pese a la "abrumadora" cantidad de asignaturas relacionadas con el diseño gráfico surgidas a partir del plan de Bolonia, el número de horas supone "sólo el aperitivo para quien muestre verdadero interés" y se antoja insuficiente "para la capacitación profesional a la que apunta el EEES" (González Díez y Núñez-Romero, 2009: 84).

A priori, pues, parece innegable la relevancia que posee el diseño gráfico en las rutinas periodísticas y, por tanto, es de suponer que el aprendizaje de estas nociones debería adquirir la misma trascendencia en una formación completa y responsable del futuro periodista. Y, sin embargo, a tenor de las dudas mencionadas, cabe preguntarse si tales necesidades están contempladas de forma rigurosa en la preparación del estudiante. De esta inicial observación derivan otros muchos interrogantes: por ejemplo, ¿en qué medida o con qué grado de profundidad es preciso abordar esta formación de cara a garantizar en el futuro una práctica periodística solvente por parte del alumno? ¿Son conscientes profesores y alumnos de la implicación de estos saberes para el desarrollo de la profesión? ¿Cuál es la verdadera presencia del diseño gráfico en el quehacer profesional? En general, ¿son suficientes las aptitudes previstas por los planes de estudio actuales?

El reto de este texto está orientado a evaluar la presencia del diseño gráfico en la formación de los estudiantes universitarios de Periodismo en España, mediante un análisis de la percepción de docentes e investigadores especializados en este campo, por una parte, y de los profesionales, en segundo lugar, en aras a descifrar si, efectivamente, los criterios académicos se ajustan a las exigencias de la profesión.

\section{Metodología}

El proyecto se basa en la combinación de dos técnicas de investigación: un análisis de datos secundarios para el estudio exploratorio (a fin de recolectar información sobre los profesores universitarios vinculados a la enseñanza del diseño gráfico en los estudios de Periodismo) y una encuesta cuantitativa, conformada por estos mismos docentes y por profesionales del ámbito periodístico. 


\subsection{Objetivo e hipótesis de trabajo}

El origen del estudio se sitúa, pues, en un afán por indagar en el estado actual de las prácticas formativas de los estudios de periodismo españoles en torno al diseño gráfico y tratar de determinar si efectivamente obedecen a los requisitos de la profesión. Como se ha insistido, más allá de los criterios objetivos establecidos en los diferentes planes de estudio, este texto pretende ahondar en las circunstancias concretas que subyacen a cada programa académico y, así, tratar de certificar su idoneidad o, al contrario, localizar posibles problemas para procurar una actividad docente más satisfactoria.

A raíz del anterior propósito, más genérico, la investigación se centra en el siguiente objetivo primordial: realizar una aproximación al escenario actual de la enseñanza del diseño gráfico en los estudios de Periodismo en España, en términos de utilidad, validez, eficacia, presencia en la industria profesional, valoración y rendimiento por parte del alumnado.

A partir de este objetivo, el trabajo plantea la siguiente hipótesis principal: "En términos generales, los estudios universitarios de Periodismo en España no ofrecen a los estudiantes una preparación adecuada en diseño gráfico, en relación a las exigencias del ámbito profesional".

Esta predicción general puede bifurcarse en un segundo nivel de hipótesis secundarias, que tratan de concretar y argumentar el sentido de la anterior:

Subhipótesis 1: La carrera de Periodismo ofrece un nivel de capacitación medio en diseño gráfico, que se interpreta como insuficiente en relación a las necesidades profesionales.

Subhipótesis 2: En los mencionados estudios, prevalecen las asignaturas vinculadas al Diseño Periodístico, cuya utilidad, unida a la de las materias relacionadas con las nuevas tecnologías, recibe la mayor valoración por parte de los estudiantes.

Subhipótesis 3: Aunque los conocimientos de diseño gráfico resultan fundamentales para la labor del futuro periodista, esta importancia no se aprecia convenientemente hasta el ejercicio de la profesión.

Subhipótesis 4: En general, el alumnado ofrece un rendimiento bajo en las materias relacionadas con el diseño gráfico.

Subhipótesis 5: Aunque un número reducido de alumnos se dedican habitualmente al ejercicio de las profesiones periodísticas más vinculadas al diseño gráfico, un gran porcentaje de estudiantes desempeñarán tareas de este tipo en algún momento de su trayectoria laboral.

\subsection{Estudio exploratorio}

Una fase inicial del proceso de investigación consistió en reunir los datos de las materias relacionadas con el diseño gráfico en los grados de Periodismo de España y de los docentes responsables de estas asignaturas (el primer grupo consultado). Para ello, siguiendo la técnica del análisis de datos secundarios, el proceso comprendió la búsqueda de la siguiente información, conforme al siguiente orden establecido:

a. Búsqueda de todos los Grados en Periodismo ofertados por las universidades españolas. 
b. Examen de los planes de estudio actuales (pertenecientes al curso 2013/2014) en las páginas web de estas facultades.

c. Selección de las asignaturas relacionadas con el diseño gráfico en estos planes de estudio, a partir del análisis de sus respectivas competencias.

d. Creación de un listado de los profesores responsables de estas asignaturas.

Bajo estas premisas, en esta primera etapa se obtuvieron los siguientes números:

- Un análisis de 35 Grados en Periodismo y 3 Grados en Comunicación del curso 2013/2014 a través de sus respectivas páginas web. Se consideró oportuno, a efectos de los objetivos de la investigación, incluir también aquellos estudios de Comunicación que ofrecen en su programa, como posible salida profesional, la incorporación a un medio informativo.

- Una relación de 88 asignaturas vinculadas con el diseño gráfico o la comunicación visual.

- Un total de $97(N)$ profesores asociados a estas materias, a los que se remitió la correspondiente invitación para participar en la investigación ${ }^{3}$.

En el caso de la relación de profesionales que formaron parte del estudio (el segundo grupo consultado), el proceso se limitó a una distribución del cuestionario a través de las redes sociales, como se especifica en el apartado relativo al proceso de investigación.

\subsection{Sujetos}

Para contrastar las predicciones anteriores, el trabajo recurrió a dos grupos principales de sujetos experimentales:

\subsubsection{Perfil docente}

Personal docente e investigador con responsabilidad en alguna asignatura relacionada con el diseño gráfico en los Grados de Periodismo durante el curso 2013-2014. La muestra final de este primer grupo estuvo compuesta por 36 profesores $(n)$ pertenecientes a los siguientes 26 diferentes centros universitarios (teniendo en cuenta que algunas de estas instituciones contaron con la participación de más de un individuo): UDIMA, Universidad de Navarra, Universidad de Málaga, Universidad Rey Juan Carlos, Universidad Complutense de Madrid, Universidad Nebrija, Universitat de Vic, Universidad de Deusto, Universitat Jaume I de Castellón, Universitat Ramón Llull, Universidad Miguel Hernández, Universidad CEU San Pablo, UCAM, Universidad CEU Cardenal Herrera, Universitat Pompeu Fabra, Universitat Autònoma de Barcelona, Universidad de Zaragoza, Universidad del País Vasco (UPV/EHU), Universidad de Castilla-La Mancha, Universitat de Valencia, Universidad de La Laguna, Univer-

3 El estudio procuró alcanzar la máxima representatividad posible mediante la consulta de los centros universitarios que ofertan los grados de Periodismo; sin embargo, no es su objeto principal realizar una enumeración de instituciones, materias o profesores, sino recoger la experiencia docente de este ámbito. Algunos componentes de la citada relación inicial se descartaron por diversos motivos, como la falta de información en sus respectivas páginas web o la escasa pertinencia final de algunas materias. 
sidade de Santiago de Compostela, Universidad de Sevilla, Universidad Carlos III, UNIR y Universidad de Murcia.

Para una mayor operatividad, en lugar de enumerar el conjunto total de asignaturas recogidas en el cuestionario (que derivaría en una relación muy vasta, debido a la heterogeneidad de sus denominaciones), se ha optado por mencionar, dentro de la segunda sección (Contenidos), la naturaleza de los conocimientos y el campo al que están adheridas.

\subsubsection{Perfil profesional}

Periodistas profesionales (en activo o no) o antiguos alumnos de licenciaturas o grados de Periodismo con algún tipo de experiencia laboral (no necesariamente especializada en diseño gráfico) y estudios finalizados en el año 2014 o anteriores.

A continuación se establece una síntesis de la información más sobresaliente sobre los perfiles de los profesionales que cumplimentaron el cuestionario, de cara a poder realizar un mejor análisis cualitativo de los datos:

- De las 88 personas ( $n$ ) finalmente encuestadas (47 mujeres y 41 hombres), un total de 75 ejercen actualmente la profesión, frente a 13 que se encuentran en desempleo, ampliando sus estudios o han desarrollado su actividad en un sector ajeno a la comunicación.

- Sobre la universidad de procedencia de los encuestados, 58 personas (un 65\%) cursaron la carrera en la Universidad Pontificia de Salamanca. Los estudios realizados por los restantes sujetos son de procedencia muy variada. Como se indica más detenidamente en el capítulo de limitaciones, el claro predominio de la citada institución constituiría el principal sesgo en la investigación de este perfil profesional.

- En relación al año en que finalizaron los estudios, se ha intentado estructurar la muestra en torno a cuatro divisiones genéricas: los más antiguos planes de estudio (finalizados en 1999 y 2004), las últimas licenciaturas (extinguidas en el año 2013) y los nuevos programas de Bolonia (que en este trabajo están representados por un número minoritario de alumnos). Se interpretó que la información sobre sus respectivos contenidos curriculares podía resultar importante en la evaluación de las hipótesis.

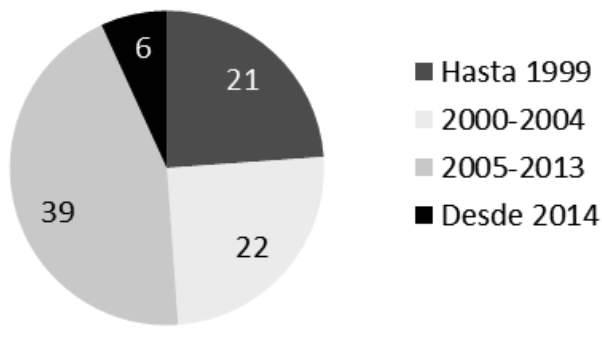

Gráfico 1: Distribución del segundo perfil (profesionales) en función del año de finalización de sus estudios 
La gran diferencia entre ambos perfiles radica en aquellas cuestiones que versan sobre los propios estudios de Periodismo: mientras el análisis de los primeros se centra en el estado actual de la formación (los denominados grados), las respuestas de los profesionales aluden, inevitablemente, y salvo contadas excepciones ( 6 sujetos) a los diferentes planes anteriores. La reciente implantación de los nuevos programas en la universidad española (cuyas primeras promociones acaban de finalizar sus estudios) exige que esta circunstancia (mencionada en las limitaciones de la investigación) sea observada con cautela en la interpretación de los datos.

\subsection{Instrumento de recogida de datos}

En relación a la segunda técnica metodológica (encuesta cuantitativa), el estudio recurre a un cuestionario formado mayoritariamente por preguntas cerradas con opciones múltiples o escalas tipo Likert en torno a una serie de cuatro grandes bloques de información generales (incluyendo un primero que se refiere al perfil de la muestra), en los que, a su vez, se integra un conjunto de variables que tratan de dar respuesta a las inquietudes reflejadas en las hipótesis y los objetivos de la investigación. Estos grandes conjuntos son los siguientes:

a. Bloque información general: trata de rescatar la información sobre cada sujeto particular; su función se limita, por tanto, a definir el perfil de la muestra. Incluye aspectos como la facultad y universidad en la que desarrolla su labor o las asignaturas que imparte (docentes e investigadores); la edad, la facultad y la universidad en la que se cursaron estos estudios, el año de finalización de la carrera, el sexo y la ocupación actual (profesionales).

b. Bloque contenidos: relacionado con la subhipótesis 2. Pretende reunir información sobre las materias ofertadas: las asignaturas vinculadas al diseño gráfico, la naturaleza de la formación que ofrecen, los ámbitos profesionales a los que están ligadas y la importancia atribuida a estas.

c. Bloque utilidad: relacionado con la subhipótesis 3 y 5 . Aborda las cuestiones relativas a la importancia del diseño gráfico en la práctica profesional, el uso de estos conocimientos en el mundo laboral (puntual o habitualmente) o la valoración de estas competencias (durante la carrera y en el mundo profesional).

d. Bloque capacitación: relacionado con la subhipótesis 1 y 4 . Se refiere a la evaluación del nivel de formación que ofrece la carrera, al medir aspectos como el rendimiento de los estudiantes en las asignaturas de diseño gráfico, el nivel de preparación que ofrece la carrera y el grado de conocimientos resultante.

Para recoger las impresiones y dibujar el mapa de la formación en diseño gráfico de los periodistas en España, se construyó un cuestionario personalizado para los dos grandes perfiles de sujetos. En las siguientes tablas se muestran todas las variables analizadas, tal y como aparecen en el instrumento de recogida de datos, agrupadas en torno a los diferentes sectores temáticos mencionados, así como los valores y niveles de cada una de ellas: 
Tabla 1. Cuestionario dirigido al personal docente e investigador de asignaturas relacionadas con el diseño gráfico en los Grados de Periodismo españoles (curso 2013/2014): bloques de información, variables o preguntas que los integran, valores y niveles

\begin{tabular}{|c|c|}
\hline Variables (preguntas) & Valores y niveles \\
\hline \multicolumn{2}{|c|}{ BLOQUE INFORMACIÓN GENERAL } \\
\hline $\begin{array}{l}\text { Facultad y universidad o filiación } \\
\text { institucional }\end{array}$ & [Pregunta abierta] \\
\hline \multicolumn{2}{|c|}{ BLOQUE CONTENIDOS } \\
\hline Asignaturas impartidas & [Pregunta abierta] \\
\hline $\begin{array}{l}\text { Tipo de formación que ofrecen estas } \\
\text { asignaturas }\end{array}$ & $\begin{array}{l}\text { - Conocimientos generales } \\
\text { - Conocimientos ligados a un ámbito profesional } \\
\text { concreto }\end{array}$ \\
\hline $\begin{array}{l}\text { Ámbitos profesionales vinculados a } \\
\text { estas asignaturas (en el caso de } \\
\text { conocimientos ligados a una disciplina } \\
\text { concreta) }\end{array}$ & $\begin{array}{l}\text { - Diseño de información en medios impresos } \\
\text { (periódicos, revistas) } \\
\text { - Diseño de páginas web } \\
\text { - Diseño en nuevos dispositivos (tablets, smartphones) } \\
\text { - Diseño audiovisual } \\
\text { - Infografía }\end{array}$ \\
\hline \multicolumn{2}{|c|}{ BLOQUE UTILIDAD } \\
\hline $\begin{array}{l}\text { Utilidad e importancia de los estudios } \\
\text { de diseño gráfico para el futuro } \\
\text { periodista }\end{array}$ & $\begin{array}{l}\text { - Es fundamental para todo periodista, más allá de su } \\
\text { especialización } \\
\text { - Es importante disponer de unos conocimientos } \\
\text { mínimos } \\
\text { - Únicamente es relevante para aquellos que quieran } \\
\text { especializarse en determinadas disciplinas }\end{array}$ \\
\hline $\begin{array}{l}\text { Valoración de la importancia del diseño } \\
\text { gráfico por parte de los alumnos } \\
\text { durante la carrera }\end{array}$ & 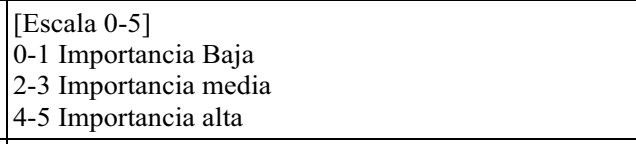 \\
\hline $\begin{array}{l}\text { Valoración de la importancia del diseño } \\
\text { gráfico por parte de los alumnos en la } \\
\text { vida profesional }\end{array}$ & $\begin{array}{l}\text { [Escala } 0-5] \\
0-1 \text { Importancia Baja } \\
\text { 2-3 Importancia media } \\
\text { 4-5 Importancia alta }\end{array}$ \\
\hline $\begin{array}{l}\text { Porcentaje de estudiantes de periodismo } \\
\text { que se dedican habitualmente al } \\
\text { ejercicio del diseño gráfico }\end{array}$ & $\begin{array}{l}\text { - Muchos }(>50 \%) \\
\text { - Bastantes }(21-50 \%) \\
\text { - Pocos }(5-20 \%) \\
\text { - Residual }(<5 \%)\end{array}$ \\
\hline $\begin{array}{l}\text { Porcentaje de estudiantes de periodismo } \\
\text { que realizan en alguna ocasión tareas de } \\
\text { diseño gráfico }\end{array}$ & $\begin{array}{l}\text { - Muchos }(>50 \%) \\
\text { - Bastantes }(21-50 \%) \\
\text { - Pocos }(5-20 \%) \\
\text { - Residual }(<5 \%)\end{array}$ \\
\hline \multicolumn{2}{|c|}{ BLOQUE CAPACITACIÓN } \\
\hline $\begin{array}{l}\text { Rendimiento del alumnado en diseño } \\
\text { gráfico durante la carrera }\end{array}$ & $\begin{array}{l}\text { [Escala } 0-5] \\
\text { 0-1 Rendimiento Bajo } \\
\text { 2-3 Rendimiento Medio } \\
\text { 4-5 Rendimiento Alto }\end{array}$ \\
\hline $\begin{array}{l}\text { Nivel de capacitación en diseño gráfico } \\
\text { que ofrece la carrera }\end{array}$ & $\begin{array}{l}\text { [Escala 0-5] } \\
\text { 0-1 Capacitación baja } \\
\text { 2-3 Capacitación media } \\
\text { 4-5 Capacitación alta } \\
\end{array}$ \\
\hline
\end{tabular}


Tabla 2. Cuestionario dirigido a profesionales o antiguos alumnos de licenciaturas o grados de Periodismo con alguna experiencia laboral: bloques de información, variables o preguntas que los integran, valores y niveles

\begin{tabular}{|c|c|}
\hline Variables (preguntas) & Valores y niveles \\
\hline \multicolumn{2}{|c|}{ BLOQUE INFORMACIÓN GENERAL } \\
\hline Edad & [Pregunta abierta] \\
\hline Facultad y Universidad & [Pregunta abierta] \\
\hline Sexo & Hombre / Mujer \\
\hline Dedicación actual & [Pregunta abierta] \\
\hline \multicolumn{2}{|c|}{ BLOQUE CONTENIDOS } \\
\hline $\begin{array}{l}\text { Materias de diseño gráfico en las que ha } \\
\text { recibido formación }\end{array}$ & $\begin{array}{l}\text { - Diseño de información en medios impresos } \\
\text { (periódicos, revistas) } \\
\text { - Diseño de páginas web } \\
\text { - Diseño en nuevos dispositivos (tablets, smartphones) } \\
\text { - Diseño audiovisual } \\
\text { - Infografía } \\
\text { - Fotografía }\end{array}$ \\
\hline $\begin{array}{l}\text { Materias de diseño gráfico que } \\
\text { considera actualmente más importantes } \\
\text { en la profesión }\end{array}$ & $\begin{array}{l}\text { - Diseño de información en medios impresos } \\
\text { (periódicos, revistas) } \\
\text { - Diseño de páginas web } \\
\text { - Diseño en nuevos dispositivos (tablets, smartphones) } \\
\text { - Diseño audiovisual (ej. posproducción) } \\
\text { - Infografía } \\
\text { - Fotografía }\end{array}$ \\
\hline \multicolumn{2}{|c|}{ BLOQUE UTILIDAD } \\
\hline $\begin{array}{l}\text { Utilidad e importancia de los estudios } \\
\text { de diseño gráfico para el futuro } \\
\text { periodista }\end{array}$ & $\begin{array}{l}\text { - Es fundamental para todo periodista saber diseñar } \\
\text { - Son importantes unos conocimientos mínimos para } \\
\text { maquetar, editar, etc. } \\
\text { - Son importantes unos conocimientos básicos para } \\
\text { hablar con los departamentos de diseño, juzgar una } \\
\text { determinada pieza, etc. } \\
\text { - No es importante: únicamente es útil para aquellos que } \\
\text { se quieran especializar en este ámbito }\end{array}$ \\
\hline $\begin{array}{l}\text { Valoración de la importancia del } \\
\text { diseño gráfico durante la carrera }\end{array}$ & $\begin{array}{l}\text { [Escala 0-5] } \\
\text { 0-1 Importancia Baja } \\
\text { 2-3 Importancia Media } \\
\text { 4-5 Importancia Alta }\end{array}$ \\
\hline $\begin{array}{l}\text { Valoración de la importancia del } \\
\text { diseño gráfico en la vida profesional }\end{array}$ & $\begin{array}{l}\text { [Escala 0-5] } \\
\text { 0-1 Importancia Baja } \\
\text { 2-3 Importancia Media } \\
\text { 4-5 Importancia Alta }\end{array}$ \\
\hline $\begin{array}{l}\text { ¿Se eligió la carrera de Periodismo } \\
\text { para dedicarse en el futuro al diseño } \\
\text { gráfico? }\end{array}$ & $\begin{array}{l}\text { - Sí, como prioridad } \\
\text { - Quizás, como una de las opciones } \\
\text { - No, en ningún caso }\end{array}$ \\
\hline
\end{tabular}

4 Esta opción se introdujo con un carácter fundamentalmente orientativo, de cara a posibles futuras investigaciones sobre este ámbito, pero no se tomó en consideración para el presente trabajo. Aunque vinculada al diseño gráfico, se interpreta que posee una envergadura suficiente para protagonizar líneas de investigación autónomas. 


\begin{tabular}{|c|c|}
\hline $\begin{array}{l}\text { ¿Se valoró dedicarse al diseño gráfico } \\
\text { una vez terminados los estudios? }\end{array}$ & $\begin{array}{l}\text { - Sí, como prioridad } \\
\text { - Quizás, como una posibilidad } \\
\text { - Quizás, como una posibilidad aunque remota } \\
\text { - Quizás, pero no como especialización sino como } \\
\text { complemento del trabajo. } \\
\text { - Se descartó por completo }\end{array}$ \\
\hline $\begin{array}{l}\text { ¿Cuál es tu relación actual con el } \\
\text { diseño gráfico? }\end{array}$ & $\begin{array}{l}\text { - Soy diseñador/a o el diseño es parte importante de mi } \\
\text { trabajo } \\
\text { - Habitualmente hago o he tenido que hacer trabajos de } \\
\text { diseño gráfico. } \\
\text { - En alguna ocasión puntual he tenido que realizar algún } \\
\text { trabajo de diseño gráfico. } \\
\text { - No soy diseñador gráfico, pero necesito de estos } \\
\text { conocimientos para trabajar en equipo con diseñadores. } \\
\text { - No tengo ninguna relación en absoluto. }\end{array}$ \\
\hline \multicolumn{2}{|c|}{ BLOQUE CAPACITACIÓN } \\
\hline $\begin{array}{l}\text { Rendimiento durante la carrera en el } \\
\text { área del diseño gráfico }\end{array}$ & \begin{tabular}{|l} 
Escala $0-5]$ \\
0-1 Rendimiento Bajo \\
2-3 Rendimiento Medio \\
4-5 Rendimiento Alto
\end{tabular} \\
\hline $\begin{array}{l}\text { Nivel de capacitación u horas en } \\
\text { diseño gráfico que ofrece la carrera }\end{array}$ & $\begin{array}{l}\text { - Muy pobre } \\
\text { - Insuficiente } \\
\text { - Suficiente } \\
\text { - Óptimo } \\
\text { - Excesivo }\end{array}$ \\
\hline $\begin{array}{l}\text { Capacidad de asumir un proyecto de } \\
\text { diseño gráfico tras los estudios }\end{array}$ & $\begin{array}{l}\text { [Escala } 0-5] \\
0-1 \text { Capacitación baja } \\
\text { 2-3 Capacitación media } \\
\text { 4-5 Capacitación alta }\end{array}$ \\
\hline
\end{tabular}

En ambos cuestionarios, igualmente, se habilitó un campo de comentarios opcional orientado a justificar las respuestas anteriores y en el que se hizo hincapié en su especial trascendencia para respaldar abiertamente las aportaciones realizadas con anterioridad. Gran parte de estos comentarios se han empleado para nutrir la discusión o reforzar las conclusiones.

\subsection{Procedimiento de investigación}

Para la distribución de los cuestionarios, el contacto del primer grupo (personal docente) se obtuvo a partir de la información rescatada en el estudio exploratorio. En el segundo conjunto (profesionales y antiguos estudiantes), al tratarse de una población de dimensiones potenciales radicalmente superiores con respecto al primero, la solicitud de participación se tramitó abiertamente a través de las redes sociales Facebook y Twitter.

Para la distribución del instrumento en ambos grupos, el estudio empleó la herramienta de cuestionarios de Google Drive, cuyos resultados fueron exportados en formato .xls (Microsoft Excel) para su medición posterior.

\section{Resultados y discusión}

Para un mejor desarrollo e interpretación de los resultados, este capítulo está estructurado según los grandes bloques de variables indicados en el anterior apartado: in- 
formación general (correspondiente al perfil de la muestra), contenidos, utilidad y capacitación.

Para la evaluación de aquellas cuestiones en las que se emplean escalas numéricas, se utilizaron dos medidas, principalmente: el valor medio (para obtener un análisis de la impresión general) y la desviación típica (como un indicador de la dispersión de las respuestas). Asimismo, en la medida de lo posible, se ha estimado conveniente la agrupación de las respuestas de ambos perfiles (docentes y profesionales) dentro de cada subsección para realizar una comparación más eficiente de los datos.

\subsection{Bloque información general (perfil de la muestra)}

Este primer bloque está orientado a reunir información sobre los sujetos experimentales para una mejor interpretación de los resultados. Estos datos aparecen ya reflejados en el epígrafe 2.3 (Sujetos).

\subsection{Bloque contenidos}

Este capítulo busca reflejar el tipo de materias impartidas por los docentes o cursadas por los actuales profesionales, así como los ámbitos profesionales vinculados y la importancia atribuida a cada uno de ellos.

Sobre el tipo de formación impartida por los docentes encuestados, existe un total equilibrio entre las dos posibilidades reflejadas en el cuestionario: los conocimientos generales de diseño gráfico y aquellos aplicados a alguna disciplina concreta. Igualmente, un gran número de sujetos (23) aborda, dentro de su responsabilidad docente, ambos tipos de conocimientos.

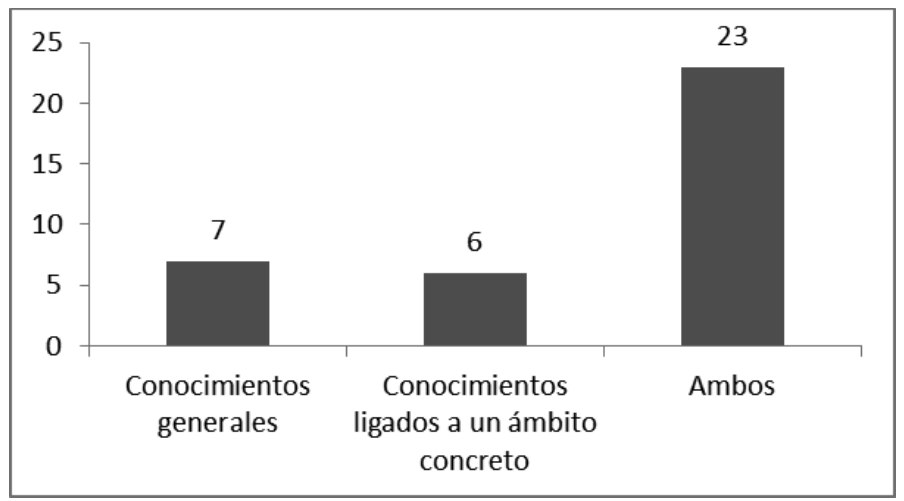

Gráfico 2. Tipos de contenidos de diseño gráfico impartidos por el primer perfil (docentes)

Por tanto, un $81 \%$ de los encuestados afirmó vincular estos conocimientos a algún tipo de ámbito profesional del diseño, frente a un 19\% restante ( 7 sujetos) cuyas materias únicamente recogen nociones generales. Respecto al primer subgrupo (individuos que imparten estudios específicos), sobresalen las materias ligadas al Diseño Periodístico (93\%), seguidas de las áreas de Infografía (62\%) y Diseño Web (37\%).

Los números anteriores están ampliamente apoyados por la respuesta del grupo de profesionales, en el que vuelve a destacar la muy sólida presencia del Diseño Perio- 
dístico en soportes impresos (92\% individuos seleccionaron esta opción). Estos datos, en todo caso, deben inevitablemente asociarse a su mayor tradición en los planes de estudio, frente a otras disciplinas que se han ido incorporando sucesivamente, en paralelo al nacimiento de las nuevas tecnologías.

En este perfil profesional, además, se preguntó a los sujetos sobre la utilidad que atribuyen a cada una de estas áreas. En este caso, los encuestados subrayan la relevancia de los conocimientos de diseño de Diseño Web (82\%), que se sitúa a una significativa distancia del Diseño de Información en soportes impresos y en los nuevos dispositivos (ambos con un 67\% de apoyo).

Sorprende, en este grupo, la especial atención que reciben la presencia y la utilidad del Diseño Audiovisual (47\% y 64\%, respectivamente) en la formación periodística, apenas citado en dos ocasiones por los docentes y cuya denominación resulta casi marginal en los programas de los centros consultados. Con el término Diseño Audiovisual se trataba de contemplar habilidades como montaje, posproducción, motion graphics, títulos de crédito o animación, algunas de ellas situadas en los límites del concepto de diseño gráfico, en sentido estricto. Se interpreta que este desfase puede deberse a varios factores: la escasa descripción de esta actividad en el cuestionario (que ha podido desorientar a los encuestados) o una cierta confusión generada en el examen de los propios descriptores de las asignaturas, que quizás condujo a su olvido parcial en el estudio exploratorio. En todo caso, constituye un imperativo inmediato indagar en las causas de este vacío.

\subsection{Bloque utilidad}

La presente sección alude a la relevancia de los conocimientos de diseño gráfico en la formación del estudiante de Periodismo en relación a las necesidades planteadas por el mercado profesional.

\subsubsection{Sobre el volumen necesario de conocimientos de diseño gráfico}

Existe un consenso absoluto por parte de los docentes en subrayar la importancia de los conocimientos de diseño gráfico más allá de la futura especialidad profesional del estudiante: un 39\% argumenta que tales nociones son esenciales en la preparación de todo periodista, frente un $61 \%$ que consideran necesarios, por lo menos, unos conocimientos básicos. Parece oportuno subrayar, en esta línea, que ninguno de los sujetos consultados asocia su utilidad exclusivamente a aquellos estudiantes que optan por especializarse en diseño gráfico.

Las respuestas del segundo grupo revelan resultados muy similares, más inclinados, si cabe, a la relevancia de unos conocimientos elementales en la profesión. Más de tres cuartas partes de la muestra ( $77 \%$ de los sujetos) coinciden en señalar esta alternativa intermedia, que, en este perfil, se ha decidido bifurcar en dos posibilidades: conocimientos básicos para poder desarrollar prototipos ya creados (maquetar un diario o actualizar una web, por ejemplo) u orientados a una relación profesional provechosa con los departamentos de diseño. Como en el primer grupo, se descarta prácticamente la última opción (relevancia reservada para las ocupaciones más específicas). 


\subsubsection{Sobre la importancia atribuida por los estudiantes}

La importancia otorgada al diseño gráfico por los estudiantes puede resultar significativa para determinar su grado de compromiso, su rendimiento en las materias, el aprendizaje posterior y, en este sentido, su posible incorporación a esta área en el futuro. En opinión del sector docente, se sugiere que esta valoración, bastante modesta en la etapa estudiantil (media de 3,1), se incrementa considerablemente durante el ejercicio de la profesión $(3,9)$, con cierto consenso en ambas (desviación típica de 1 y 0,7 , respectivamente). En sus comentarios, además, muchos profesores se lamentan, en este punto, del exclusivo apego a las herramientas tecnológicas (en detrimento de una mayor valoración de la formación teórica), de la escasa cultura inicial del alumno en relación a este ámbito y, en general, de la falta de comprensión sobre la verdadera implicación del diseño en las labores periodísticas.

En la misma línea que los anteriores, la valoración de los profesionales experimenta un notable crecimiento durante el desempeño de la profesión frente al período universitario. Sin embargo, en relación a los docentes, aunque la dispersión es ligeramente mayor (desviación típica de 1,3 y 0,9 ) esta apreciación se incrementa de forma considerable en ambas etapas (media de 3,5 y 4,3, respectivamente), a la vez que sitúa sus respuestas muy cerca de los valores máximos de la escala y permite, por tanto, certificar la relevancia de estos estudios en el mundo laboral.

\subsubsection{Sobre el número de alumnos que se dedican al diseño gráfico}

Una estimación aproximada del volumen de alumnos que acaba asumiendo habitual u ocasionalmente tareas de diseño gráfico puede constituir un indicador de sumo interés para medir la relevancia de esta materia. En este sentido, pese a que no existe un consenso explícito sobre la cantidad de estudiantes que acaban desempeñando de forma continuada actividades de esta naturaleza, sí existe un acuerdo absoluto en los profesores (33 sujetos) en que muchos periodistas (interpretado aquí como un porcentaje superior al 50\%) deben asumir en alguna ocasión tareas de diseño gráfico en su vida profesional.

Para contrastar los datos del primer grupo, se sustituyó, en el cuestionario dirigido a los profesionales, la anterior escala por un conjunto de alternativas más amplio, con la intención de reflejar de forma más precisa la situación actual de cada individuo y describir más pormenorizadamente la esencia de estas competencias. En este nuevo abanico, un número minoritario afirma que sus tareas cotidianas no tienen ninguna relación con el diseño gráfico; el resto (un 91\%) reconoce que estos quehaceres tienen en su trabajo cotidiano una aplicación frecuente (como profesionales o como diseñadores habituales) o puntual (como diseñadores ocasionales o en su relación diaria con los departamentos gráficos). Estos últimos sectores, señalados por 54 individuos, son los que obtienen un respaldo mayoritario.

Otro aspecto que puede resultar interesante de cara a interpretar la realidad actual de las tareas de diseño en la industria periodística son las expectativas del estudiante potencial. Como se ha indicado, se presume un cierto desconocimiento o una escasa vocación inicial del futuro alumno de Periodismo hacia el ámbito del diseño gráfico. En efecto, una amplia mayoría admite un peso inexistente del diseño gráfico en la se- 
lección de la carrera de Periodismo. Un posible reflejo de la mencionada ignorancia inicial se debe a la importante variación de estos números tras cursar estos estudios: solo un $20 \%$ (frente al $69 \%$ inicial) descarta totalmente esta posibilidad y, aunque las preferencias claras hacia este ámbito siguen siendo escasas (7\%), se multiplican aquellos casos que pasan a considerarlo como una alternativa laboral más (73\%). Esta cifra resulta de sumar los estudiantes que conciben el diseño como una opción (firme o más improbable) y los que contemplan esta actividad como un complemento de su trabajo.

\subsection{Bloque capacitación}

El último gran bloque del estudio está asociado al nivel de preparación que ofrece la carrera de periodismo o al aprovechamiento de esta formación por parte del alumnado.

\subsubsection{Sobre el nivel de preparación en diseño gráfico de la carrera}

Uno de los grandes objetivos del estudio se refiere al nivel de preparación en diseño gráfico que ofrece la carrera de Periodismo, en relación a las necesidades del mercado. En la pregunta más directa sobre esta cuestión, las respuestas de los docentes hablan de un nivel más bien modesto, con una cifra ligeramente más alta que la media $(2,77)$ y una percepción más bien desigual entre los participantes, a tenor de lo indicado por la desviación estándar $(1,19)$.

De forma más contundente, el segundo grupo consultado corrobora los resultados del primero, un descontento que avalan las cifras obtenidas en este apartado: tan solo un $22 \%$ se muestra conforme con la formación recibida en esta área, y, de estos, únicamente un 5\% la califica de "óptima". El resto (78\%) tilda esta experiencia de "muy pobre" o "insuficiente"5.

Para reforzar esta variable, se introdujo como criterio adicional la capacidad de los sujetos de asumir con solvencia un proyecto de diseño, que se interpreta como un supuesto síntoma de los conocimientos adquiridos. La respuesta es, en este punto, absolutamente heterogénea y variable, y alcanza enormes similitudes en todas las opciones ofrecidas (0-5). Al contrastar estos datos con la anterior escala, cabe intuir que la mejora en las aptitudes en algunos casos obedece a una hipotética formación posterior, bien a través de nuevos estudios o bien a raíz del ingreso en la vida profesional.

\subsubsection{Sobre el rendimiento de los estudiantes en diseño gráfico}

Pese a los discretos resultados y a las dificultades mencionadas en el nivel de preparación de la carrera, el rendimiento de los estudiantes en las materias de diseño gráfico se describe como moderadamente satisfactorio (media de 3,4), según la percepción de los docentes: no se registra apreciación alguna en los dos valores inferiores $\mathrm{y}$, sin embargo, más de la mitad (55\%) coincide en calificarlo de "alto" o "muy alto".

Esta impresión docente sobre una tibia satisfacción hacia el rendimiento de los estudiantes está respaldada por estos con un promedio de 3 puntos; en este caso, sin em-

5 En este caso, se ha sustituido la escala numérica por un conjunto de 5 alternativas en cuyo significado se trataba explícitamente de hacer hincapié, como la última opción ("excesivas"), pese a que finalmente un único sujeto se inclinó por esta opción. 
bargo la diversidad es muy superior (desviación estándar de 1,3). La razón parece evidente: mientras las valoraciones generales tienden a rechazar los extremos, las calificaciones personales incorporan una mayor precisión en el juicio.

\section{Conclusiones}

Tras la observación y análisis de los resultados obtenidos, este capítulo recoge las conclusiones finales respecto a los objetivos e hipótesis formulados en el origen de este estudio. Es imprescindible interpretar siempre estas conclusiones a partir de las impresiones de los sujetos experimentales, destacadas en el capítulo anterior:

1. Las aportaciones de los estudios de Periodismo en España respecto al ámbito del diseño gráfico son insuficientes en relación a las demandas de la profesión.

En general, los resultados evidencian un consenso importante entre académicos y profesionales en señalar que las enseñanzas en materia de diseño gráfico son escasas, aunque esta apreciación es más rotunda y homogénea en el caso del segundo grupo, notablemente más crítico. Se estima que el motivo de esta desviación puede residir en las mejores condiciones ofrecidas por los nuevos grados (analizados por los docentes), frente a los antiguos planes (evaluados por la mayor parte de los profesionales encuestados). Otros indicadores, como la desigual capacidad para desarrollar tareas de diseño gráfico una vez finalizada la carrera, parecen fortalecer la discreta percepción general.

2. Los estudios de Periodismo ofrecen un nivel de capacitación medio en diseño gráfico, pero estos conocimientos se tornan insuficientes en relación a la realidad profesional.

Esta conclusión respalda la primera subhipótesis del estudio, al reflejar que la cantidad de horas, las asignaturas o la profundidad en el aprendizaje del diseño gráfico resultan escasas y que, por tanto, no responden a las exigencias del mercado profesional y a su demanda actual de habilidades multidisciplinares. Esta sensación se acentúa en el ámbito profesional, merced, quizás, a la posibilidad de definir verbalmente (y no de forma numérica) en el cuestionario el alcance de los estudios. Esta percepción es, además, altamente homogénea y no sufre altibajos importantes en función de los planes de estudio cursados.

3. En un contexto de equilibrio casi absoluto entre el número de materias generales y específicas de diseño gráfico, destaca la presencia del Diseño Periodístico Impreso en los estudios, aunque se incrementa, por parte de los profesionales, la valoración de las asignaturas relacionadas con las nuevas tecnologías.

Entre las asignaturas de los docentes encuestados, la envergadura de los conocimientos generales y específicos de diseño gráfico es casi idéntico. Entre estos últimos, el Diseño Periodístico en soportes impresos está universalmente presente en la práctica totalidad de las propuestas formativas; junto a este ámbito, los actuales profesionales conceden actualmente una gran importancia a los conocimientos relacionados con el medio audiovisual, los nuevos dispositivos y, especialmente, el diseño web, probablemente motivados por el cambio de paradigma tecnológico, la menor atención recibida por sus respectivos estudios o una cierta cautela ante las dificultades que atraviesa el papel. 
4. Las nociones de diseño gráfico resultan de gran relevancia en las tareas propias del periodista, pero esta percepción se adquiere, fundamentalmente, en la vida profesional.

Docentes y profesionales señalan que es preciso disponer, por lo menos, de unos conocimientos mínimos de diseño gráfico y que estos resultan trascendentales para un eficiente ejercicio profesional. Sin embargo, esta utilidad, relativizada durante la etapa académica, adquiere más protagonismo y prestigio durante la práctica profesional, transformación que se atribuye a un elevado desconocimiento de los estudiantes sobre la verdadera función del diseño y la realidad del mundo laboral.

5. En general, el alumnado ofrece un rendimiento moderadamente satisfactorio en las asignaturas relacionadas con el diseño gráfico.

A pesar de que apenas un número testimonial de estudiantes afirma haber cursado la carrera de Periodismo para dedicarse preferentemente a actividades ligadas al diseño gráfico (cuestión que sitúa estos conocimientos lejos de sus intereses originales), profesores y profesionales valoran razonablemente su rendimiento. En el caso de estos últimos, la heterogeneidad de las respuestas es mucho mayor (debido, probablemente, a la imprecisión que comportan los juicios globales de los primeros).

6. Aunque solo un grupo reducido de periodistas se dedica habitualmente al ejercicio del diseño gráfico, la gran mayoría de los profesionales precisan de estos conocimientos en sus labores cotidianas o en algún momento de su profesión.

El número de estudiantes que selecciona los estudios de Periodismo para especializarse en el área del diseño gráfico es, pues, casi anecdótico; aunque aumentan tras cursar los estudios, estas preferencias siguen siendo escasas. Frente a esta realidad, la inmensa mayoría reconoce haber recurrido a estos conocimientos en algún momento de su vida profesional (por lo menos), afirmación respaldada por los docentes del área.

\section{Limitaciones y líneas futuras de investigación}

Las futuras líneas de investigación con las que se pretende profundizar en este trabajo (en las que, asimismo, se recogen sus principales limitaciones) son las siguientes:

1. Contrastar los resultados obtenidos con las apreciaciones de los estudiantes de Periodismo con matrícula en vigor. Se valora que el tiempo transcurrido desde la finalización de los estudios puede desvirtuar las impresiones y, por tanto, contaminar los resultados sobre factores como el rendimiento y las perspectivas. Asimismo, puede resultar interesante recoger y comparar estas reflexiones con la opinión de docentes e investigadores menos afines al ámbito del diseño gráfico.

2. Enriquecer la muestra, especialmente en relación con los miembros del ámbito profesional, debido a las excesivas similitudes que se aprecian en algunos parámetros (como el relativo al centro de procedencia, que es el más evidente). Por otra parte, es un deber urgente tratar de suplir la que, como ya se ha indicado, constituya quizás la principal debilidad de la investigación (aunque, en estos momentos, se trate de una dificultad coyuntural): restringir, en el caso de los profesionales, el concepto de "tipo de estudios cursado" a aquellos surgidos al amparo del Espacio Europeo de Educación Superior y valorar únicamente estos programas. 
3. Contrastar las conclusiones con un informe cuantitativo en profundidad, de carácter objetivo, sobre la situación del diseño gráfico en los grados de Periodismo: alcance de la oferta, nombre de las asignaturas, créditos, curso en el que se integran o contenidos curriculares, similar al desarrollado hace algún tiempo por Muñoz Bécares (2006) sobre la presencia del Diseño Periodístico en los estudios de licenciatura.

4. Perfeccionar el instrumento de recogida de datos, a raíz de las múltiples aportaciones, de gran interés, vertidas en el cuestionario por los propios participantes, y desde la necesidad de reforzar y ampliar la información de los diferentes capítulos.

5. Hacer énfasis en el análisis sobre algunas facetas aquí mencionadas pero escasamente desarrolladas (como la fotografía o el ámbito audiovisual), reflexionar sobre la intervención de determinados fundamentos, en concreto (la tipografía, por ejemplo) o desarrollar estudios similares que arrojen luz sobre la presencia del diseño gráfico en otras parcelas universitarias de la comunicación (Publicidad o Comunicación Audiovisual, por ejemplo), que, como se ha evidenciado en varias fases de este estudio, poseen un lazo muy estrecho con las tareas propias del periodismo.

\section{Referencias bibliográficas}

ANECA (2005): Libro blanco. Títulos de grado en Ciencias de la Comunicación. Madrid, Agencia Nacional de Evaluación de la Calidad y Acreditación (ANECA): http://www.aneca.es/media/150336/libroblanco_comunicacion_def.pdf [Consulta: 11 de julio de 2014].

BENITO, Mario (2011): "Maquetar deportes", en Encajabaja: prensa y diseño: http://www.encajabaja.com/2011/03/maquetar-deportes.html [Consulta: 1 de julio de 2014].

CARDOSO, Paulo Ribeiro; COSTA, Francisco; VERÍSSIMO, José Domingos; CORREIA, Maria do Rosário; y MARQUES, Gisela (2011): “O perfil dos licenciados en Publicidade em Portugal. Uma perspectiva dos estudantes, dos professores e dos profissionais". Questiones Publicitarias, Vol. I, N¹6, pp. 1-23.

CASASÚS, Josep María (1998): Ideología y análisis de medios de comunicación (4º ed.). Barcelona, C.I.M.S.

CONTRERAS, Fernando y SAN NICOLÁS, César (2001): Diseño gráfico, creatividad y comunicación. Madrid, Blur Ediciones.

DE PABLOS, José Manuel (2003): Tipografía para periodistas. Madrid, Universitas.

FRECHETTE, Casey (2013). "What jounalists need to know about Web design", en Poynter.org: http://www.poynter.org/how-tos/newsgathering-storytelling/visualvoice/206287/what-journalists-need-to-know-about-web-design/ [Consulta: 23 de mayo de 2014].

GARCÍA YRUELA, Jesús (1991): "Introducción a la tecnología de la información en periodismo impreso", en BARTOLOMÉ, Donaciano; GARCÍA YRUELA, Jesús; DE PABLOS, José Manuel et al.: Estudios sobre tecnologías de la información. Madrid, Sanz y Torres, pp. 65-108.

GONZÁLEZ DÍEZ, Laura y NÚÑEZ-ROMERO, Francisco (2009): “La enseñanza universitaria del Diseño Periodístico como vehículo para la inserción en el mercado 
laboral", en PÉREZ CUADRADO, Pedro y PUEBLA, Belén (coords.): Cómo se configura el mercado laboral. Madrid, Servicio de Publicaciones de la URJC, pp. 75-86: http://eciencia.urjc.es/bitstream/10115/11309/3/libro\%201\%20repositorio.pdf [Consulta: 9 de mayo de 2014].

GONZÁLEZ SOLAS, Javier (2001): "Los límites de la significación en la Identidad Visual Corporativa", en Area Abierta, 1: http://dialnet.unirioja.es/servlet/articulo? codigo $=1354472$ \&orden=1\&info=link [Consulta: 23 de noviembre de 2012]. MUÑOZ BÉCARES, Amor (2006): La docencia del Diseño Periodístico en España. Valencia, Universidad CEU Cardenal Herrera.

PÉREZ CUADRADO, Pedro (2009): "Una aproximación al Diseño de Información en las Facultades de Comunicación a punto de concluir el proceso de Bolonia”, en Diseño In [forma]: http://disenourjc.blogspot.com.es/2009/10/una-aproximacional-diseno-de-la.html [Consulta: 9 de mayo de 2014].

ROM, Josep (2002): Els fonaments del Disseny Gráfic. Barcelona, Trípodos.

SALAVERRÍA, Ramón y BARRERA, Carlos (2009): "The Spanish Journalism Education Landscape", en TERZIS, Georgios (ed.): European Journalism Education. Chicago, Intellect Books Ltd, pp. 319-330: http://dspace.unav.es/dspace/bitstream/10171/7264/1/European_Journalism_Education_SPAIN_pp319-330.pdf [Consulta: 29 de julio de 2014]. 